\title{
Prospective audit comparing ambulatory day surgery with inpatient surgery for treating cataracts
}

\author{
S P B Percival, S S Setty
}

\begin{abstract}
Objectives - To compare the cost effectiveness and safety of inpatient cataract surgery (with one night in hospital postoperatively) with ambulatory day case surgery under local anaesthesia.

Design - Prospective study of patients receiving inpatient (group 1) or day case (group 2) surgery.
\end{abstract}

Setting - One ophthalmic surgical firm.

Patients - 100 patients in each group, excluding those with coexisting ocular conditions, contraindications to local or request for general anaesthesia, ill health, or lack of agreed minimum social care; four patients died during follow up.

Interventions - Envelope method and implantation of the posterior chamber lens into the capsular sac in both groups.

Main measures - Perioperative complications, operating and turnover times, visual outcome at three to six days and 10 weeks to six months after operation, patient satisfaction (according to self administered questionnaire) at three to six days, and total costs (1989 salaries) for both groups.

Results - Patients in both groups did not differ significantly in age or sex, perioperative complications, visual outcome $(6 / 9$ or better in 78 patients in group 1 and 75 in group 2 at one month after operation and $6 / 12$ or better in $92 / 98$ in group 1, 90/98 in group 2 at final follow up), or patient satisfaction. The mean total cost per patient for group 1 patients was $£ 365.99$ and for group $2, £ 221.62$.

Conclusions - Day case surgery for cataract is safe and more cost effective.

Implications - Day case surgery should be recommended to increase availability of cataract surgery and thereby improve quality of life for more patients.

Ophartment of Scarborough District Hospital, Yorkshire YO12 6QL YO12 6QL
S P B Percival consultant ophthalmic surgeon

S S Setty, senior house officer

Correspondence to: $\mathrm{Mr} \mathrm{S}$ Percival

Accepted for publication 16 December 1991. behind other European countries in the move towards day case cataract surgery under local anaesthesia: the percentage of day cases $(5.7 \%)$ was less than half that in Belgium, the Netherlands, Denmark, Spain, France, and Sweden, and the percentage of operations performed under local anaesthesia (27.1\%) was less than a third of that in Germany, Denmark, France, Italy, and Sweden. ${ }^{1}$

In 1989 we carried out a pilot study in which 60 patients were randomised into three groups: receiving general anaesthesia as an inpatient, local anaesthesia as an inpatient, and local anaesthesia as a day patient. All patients had agreed to any one of the three options when lised for surgery. The results suggested that local anaesthesia was more cost effective and safer for the patient than general anaesthesia and significantly reduced total theatre time per patient. The cost of materials for general anaesthesia was fifteen times that for local anaesthesia, and the staff cost per patient double. Outpatient surgery was more cost effective than inpatient surgery, but the numbers of patients were too few to verify its safety. ${ }^{2}$

Our aim was to compare the cost effectiveness and safety of inpatient cataract surgery with ambulatory day case surgery under local anaesthesia in a prospectively randomised group of patients.

Patients and methods

In April 1989 we set up a study to compare 100 cataract operations performed as inpatient admissions with 100 performed as ambulatory day cases, all under local anaesthesia. The last operation to be entered into the study was performed in July 1990. In the intervening period all patients listed for cataract operations by one surgeon (SPBP) were asked if they would accept either a day case or inpatient option. Agreement to either option was marked on the waiting list card as was any reason for potential exclusion from the study.

For day case surgery agreement included minimum social care by a relative or friend (name and telephone number given in writing) to supervise convalescence, ensure the application of drops, arrange transport, and escort the patient to and from hospital on the day of operation and to hospital for the first follow up visit. Leaflets were issued to each patient giving instructions on the necessary procedure before surgery, recommended activities after surgery, and possible symptoms that might be expected.

Exclusion criteria were $(a)$ coexisting ocular 
conditions such as ocular hypertension or uveitis, (b) a contraindication to local anaesthesia, such as extreme anxiety or senile dementia, (c) a specific request for general anaesthesia, (d) ill health (for example, inability to lie flat or experience of dyspnoea at rest), and (e) inability to ensure agreed minimum social care.

The first 200 patients listed who were suitable for the study were randomly allocated to two groups to receive cataract surgery under local anaesthesia with one postoperative night in hospital (group 1) and ambulatory surgery under local anaesthesia (without premedication) in a day case theatre (group 2). Once randomised, patients could not switch groups. Patients who were withdrawn were replaced by other randomised patients taken consecutively from the randomised lists until a total of 100 consecutive patients had been operated on in each group.

All patients were clerked by the senior house officer, including a basic examination of cardiac and respiratory functions. Group 1 patients were examined on the day before surgery if they were on a morning list and were included among a mixed list of opthalmic cases. Group 2 patients were asked to attend at $830 \mathrm{am}$; lists started at $930 \mathrm{am}$ and generally comprised six patients. Group 1 patients received oral temazepam $10 \mathrm{mg}$ one hour before surgery; group 2 patients received no premedication. Local anaesthesia comprised topical oxybuprocaine and a peribulbar block but no facial block. ${ }^{3}$ Oculocompression was maintained for 20 minutes or for the duration of the preceding operation. Each operation was performed by the same surgeon (SPBP) with the following assistants: one scrubbed theatre sister (grade $G$ ), one runner nurse (grade $\mathrm{A}$ ), and one theatre technician who supervised the use of an oximeter and piped oxygen under the towelling to the patient's face. For the inpatient theatre (group 1) there was additionally one senior house officer in ophthalmology who attended the session for teaching purposes as part of the weekly routine and gave each peribulbar block, one porter for transportation by trolley, and one ward nurse (grade C) who stayed with the patient throughout the procedure. The ward nurse rotated with other ward nurses as advised by the nursing administration in order to gain work experience but was not made available for the day case theatre. For the day case theatre (group 2) SPBP gave the peribulbar blocks between patients as a senior house officer was unavailable for these sessions, the theatre technician sat with the patient, and the patient was transported in a wheelchair without the need for a porter. Operations were carried out specifically without a standby anaesthetist, although for group 1 patients anaesthetists working in adjacent rooms were available if necessary.

The operative technique was the same in each patient using the envelope method and implantation of a posterior chamber lens with intent into the capsular sac. ${ }^{3}$ All eyes were implanted with a single piece looped lens into the posterior chamber, except for one case of zonular rupture that required an anterior chamber lens. The viscoelastic sodium hyaluronate was used only when it contributed to the safety of the operation. As it is associated with raised intraocular pressure postoperatively it was routinely aspirated from the anterior chamber before the close of surgery, except when precluded by a threat of vitreous in the anterior chamber. Topical metipranolol was applied at the close of surgery, but routine acetazolamide was not used.

After operation patients in group 1 were returned to the ward for bed rest. A senior house officer undertook slit lamp examination and tonometry four to six hours after operation and examined the eyes again the following morning before discharge. Patients in group 2 were fully mobilised after operation and waited either in the "overnight stay" ward adjacent to the outpatient theatre suite or in a ward day room. Slip lamp examination and tonometry were performed as before, and the patient was discharged or admitted for one night if there was doubt about progress. Patients in this group considered by SPBP to be at risk of developing postoperative problems were re-examined by him one day after surgery. It was also explained that patients could telephone the senior house officer on call if they were in the least concerned about their progress at any stage or could present at the eye clinic on any weekday morning. General practitioners were informed in a discharge letter of the procedures carried out. All patients were re-examined by a senior house officer three to six days after operation.

Details of each patient were collated on computer compatible proformas issued at the time of surgery and completed concurrently with postoperative examinations. They included $(a)$ perioperative complications, use of sodium hyaluronate, operating time (speculum in to speculum out), theatre time (time previous patient left theatre or start of list to time patient left theatre); (b) results of examination three to six days after operation; (c) number of days in hospital and reasons for extended stay; (d) assessment of ocular or systemic adverse reactions; (e) response to a questionnaire three to six days after operation which asked whether the arrangements for admission were convenient, the operation was painful, and the same method would be preferred for the other eye; and $(f)$ results of refraction and slip lamp examination one month after operation.

Postoperative examinations were made in the outpatient clinic by a senior house officer, the clinical assistant, or SPBP, according to availability and appropriateness. Final visual outcome was assessed between 10 weeks and six months after operation.

The cost of materials, staff time, ward stay, and equipment was analysed with information from the pharmacy and supplies, personnel, and finance departments. Statistical analysis was by Student's $t$ test (Northwick Park 
Hospital Routine Statistical Package) and the Confidence Interval Analysis Microcomputer Program on an Amstrad PC 1640.

\section{Results}

POTENTIAL FOR DAY SURGERY

A total of 480 patients were listed for cataract surgery during the 15 month study period; two were unsuitable for local anaesthesia, 29 were unsuitable for day surgery because of coexisting ocular conditions, 16 specifically requested a general anaesthetic, 38 requested inpatient surgery for social reasons, and 395 $(82 \%)$ accepted the possible option for day surgery, agreeing to provide their own transport. Of these 395, only the first 212 were included in the randomised study, 12 of whom were withdrawn (table 1).

The mean age of patients in group 1 was 75.0 and in group 2, 75.8 (range 48-95). There was no significant age or sex variation between the groups. Medical problems included hypertension, cardiac failure, diabetes mellitus, cerebrovascular disease, asthmatic bronchitis, and long term steroid treatment. In no patient after medical clerking was it considered necessary to postpone surgery, and no patient caused anxiety about cardiorespiratory function during surgery.

SURGICAI COMPIICATIONS

Table 2 lists the operative complications; there was no significant difference between the two groups. Table 3 shows the reasons for extended stay. In group 1 only one patient required an extended stay medically, owing to iris prolapse. However, this may not have been the result of a malsutured wound and requires explanation. At operation the following day a

Table 1 Reasons for withdrawal of 12 patients from cataract operations

\begin{tabular}{lcc}
\hline Reason & Group 1 & Group 2 \\
\hline Patient ill & 3 & 3 \\
$\begin{array}{l}\text { Group 1 entry requested for social } \\
\text { reasons }\end{array}$ & 0 & 2 \\
$\begin{array}{l}\text { Patient refused admission, wanted } \\
\text { entry to group 2 }\end{array}$ & 1 & 0 \\
$\begin{array}{l}\text { Patient decided on private treatment } \\
\text { Patient on holiday }\end{array}$ & 0 & 1 \\
Patient requested general anaesthesia & 0 & 1 \\
\hline
\end{tabular}

Table 2 Operative and postoperative complications

\begin{tabular}{|c|c|c|}
\hline Complication & $\begin{array}{l}\text { Group } 1 \\
(n=100)\end{array}$ & $\begin{array}{l}\text { Group } 2 \\
(n=100)\end{array}$ \\
\hline \multicolumn{3}{|l|}{ Operative } \\
\hline $\begin{array}{l}\text { Iridotomy or sphincterotomy for } \\
\text { small pupil }\end{array}$ & 3 & 3 \\
\hline Posterior capsule rupture & 9 & 8 \\
\hline Vitreous loss & 1 & 1 \\
\hline Zonule rupture & 0 & 3 \\
\hline Scleral collapse & 0 & 1 \\
\hline Significant hyphaema & 1 & 0 \\
\hline \multicolumn{3}{|l|}{ Postoperative } \\
\hline Iris prolapse & 1 & 1 \\
\hline Corneal oedema at first visit & 1 & 0 \\
\hline $\begin{array}{l}\text { Intraocular pressure }<23 \mathrm{~mm} \mathrm{Hg} \text { at } \\
\text { first visit }\end{array}$ & 0 & 2 \\
\hline Pupillary fibrin reaction at first visit & 1 & 0 \\
\hline $\begin{array}{l}\text { Superior branch retinal artery } \\
\text { occlusion (one day after surgery) }\end{array}$ & 0 & 1 \\
\hline Non-circular pupil at one month & 5 & 4 \\
\hline Vitreous in anterior chamber & 1 & 4 \\
\hline Macular oedema at one month & 2 & 3 \\
\hline Active uveitis at one month & 1 & 0 \\
\hline Optic atrophy & 1 & 0 \\
\hline
\end{tabular}

hard eye was found which required a posterior sclerotomy before additional sutures could be placed and the anterior chamber reformed. The patient was a hypertensive 83 year old, who later developed optic atrophy and had a visual acuity of only hand movements. The retrospective diagnosis was retinovascular insufficiency with a probable choroidal haemorrhage of delayed onset soon after operation.

In group 2, five patients were admitted for one night, one because of a severe punctate epitheliopaphy; one because of slight shallowing of the anterior chamber; and three because of intraocular pressure $>24 \mathrm{~mm} \mathrm{Hg}$. In these three sodium hyaluronate had been used during operation: two patients had had it aspirated without further treatment; in the other the eye was complicated by posterior capsule rupture, sodium hyaluronate had not been aspirated, but topical metipranolol had been given at the close of the operation followed by intramuscular acetazolamide 500 mg. A sixth patient had developed sudden blurring on the second day after operation and was found to have an iris prolapse when she attended the outpatient clinic the following morning.

VISUAL, OUTCOME AT ONE MONTH

Seventy eight patients in group 1 and 75 of those in group 2 had a visual acuity of $6 / 9$ or better when refracted one month after operation. Twelve patients in each group had visual acuity $<6 / 12,11$ because of macular degeneration, five because of macular oedema (which later resolved spontaneously in four), two because of capsular fibrosis, and one each because of a corneal epithelial deficit, corneal oedema after grafting, high astigmatism, diabetic retinopathy, branch retinal artery occlusion, and optic atrophy.

FINAL VISUAL OUTCOME

At the final assessment two patients in each group had died, leaving 196, of whom, 92 patients in group 1 and 90 in group 2 could see $6 / 12$ or better. If pre-existing conditions such as macular degeneration (11 patients) and diabetic retinopathy (one) are excluded the incidence of patients seeing $6 / 12$ or better was $92 / 94(97 \%)$ in group 1 and $90 / 90$ $(100 \%)$ in group 2 . The two group 1 patients unable to see $6 / 12$ as the result of operation had persistent macular oedema (visual acuity $6 / 18$ ) or optic atrophy associated with iris prolapse (visual acuity limited to hand movements) respectively.

PATIENT PREFERENCE

In answer to the questionnaire on patient

Table 3 Number of days of extended inpatient stay and reason

\begin{tabular}{lcc}
\hline Reason for stay & Group 1 & Group 2 \\
\hline Raised intraocular pressure $(\mathrm{n}=3)$ & 0 & 3 \\
Iris prolapse $(\mathrm{n}=2)$ & 2 & $1^{\star}$ \\
Social reasons $(\mathrm{n}=1)$ & 2 & 0 \\
Anterior chamber shallow $(\mathrm{n}=1)$ & 0 & 1 \\
Punctate epithelial keratopathy $(\mathrm{n}=1)$ & 0 & 1 \\
\hline
\end{tabular}

*Admission for one night on the third postoperative day. 
Table 4 Material costs per operation

\begin{tabular}{lr}
\hline Item & \multicolumn{1}{c}{$£$} \\
\hline $\begin{array}{l}\text { Routine preoperative and postoperative drops, } \\
\text { intramuscular antibiotic, dressings, and dark }\end{array}$ & 4.62 \\
glasses & \\
Anaesthetic agents, needles, and syringes & 2.07 \\
Wear and tear and servicing of microscope & 2.45 \\
Wear and tear of diamonds and non-disposable & 3.00 \\
equipment & \\
$\begin{array}{l}\text { Disposable tubing, sutures, cannulas, cystitomes, } \\
\text { and gloves }\end{array}$ & 39.09 \\
Hartman's irrigating solution (1 litre/list) & 0.17 \\
Subconjunctival framycetin 250 mg/betnesol $2 \mathrm{mg}$ & 4.04 \\
Posterior chamber lens implant & 42.00 \\
Viscoelastics (sodium hyaluronate) mean & 17.65 \\
\hline Total & 115.09 \\
\hline
\end{tabular}

^Sodium hyaluronate was used for 37 patients in group 1 and 37 in group 2.

satisfaction, patients in group 2 seemed to be entirely satisfied: all stated that arrangements for admission were convenient, the operation was painless, and that they would prefer the same method for the other eye. In group 1, two patients stated that they would have preferred day surgery and two that the operation was painful.

costs

The mean material cost of each operation was $£ 115.09$ (table 4). Figures were not available for the cost of using the theatre sterile supplies unit or for the laundry of linen in theatre. Table 5 shows staff costs.

Table 5 Cost of staff/hour*

\begin{tabular}{lr}
\hline Staff grade & \multicolumn{1}{c}{$£$} \\
\hline Consultant surgeon & 19.77 \\
Senior house officer & 8.30 \\
Theatre sister & 8.47 \\
Theatre runner & 3.59 \\
Ward nurse & 4.94 \\
Theatre technician & 5.75 \\
Porter & 3.11 \\
\hline
\end{tabular}

${ }^{\star}$ Calculated from mid-1989 salaries (11 sessions) divided by 52.143 weeks and by 40 hours/week.

There was no significant difference in operating time between the two groups. There was a difference, however, in length of time spent in theatre and mean staff cost per patient (table 6). In group 2, for which there was no porter, no senior house officer, no ward nurse, and for which the surgeon gave all peribulbar blocks, changeover time between patients was significantly greater but at a reduction in cost of staff per patient.

The costs of hospital stay (ward nursing, laundry, catering) were calculated at $£ 121.84 / 24$ hours' inpatient stay and $£ 87.46$ for a day case stay. Eighty one patients in group 1 had their operation performed during a morning list and required hospital admission the previous day and therefore a two day

Table 6 Theatre time (minutes) and staff cost per patient $(£)$

\begin{tabular}{|c|c|c|}
\hline & Group 1 & Group 2 \\
\hline $\begin{array}{l}\text { Mean (SD) operating time } \\
(95 \% \text { confidence interval })\end{array}$ & $17.01(3.47)(16.32$ to 17.70$)$ & $16.25(4.41)(15.38$ to 17.12$)$ \\
\hline $\begin{array}{l}\text { Mean (SD) changeover time } \\
(95 \% \text { confidence interval) }\end{array}$ & $8.66^{\star}(2.25)(8.21$ to 9.11$)$ & $10.91^{\star}(2.03)(10.51$ to 11.31$)$ \\
\hline Mean total theatre time & 25.67 & 27.16 \\
\hline Mean staff cost per patient $\dagger$ & 23.06 & 17.01 \\
\hline
\end{tabular}

admission period. Two of these patients had their stay extended by two nights each, one for social reasons, the other because of iris prolapse. Nineteen patients in the group were operated on during an afternoon list and required admission for only one night. The days in hospital for patients in group 1 should have totalled 187, but two patients were inadvertently discharged by a nurse on the day of operation along with day cases.

Table 7 shows a summary of costs for the 100 patients in each group.

\section{Discussion}

This study compares the complications, visual outcome, and costs of ambulatory care with inpatient care for cataract surgery under local anaesthesia. Cataract surgery is never completely safe, but as the same techniques were used in each group it was not surprising that complication rates were similar. The question is whether without overnight hospital care and immediate examination the following morning patients are at risk of a compromised visual outcome. We believe that they are not, provided that surgery takes place in a dedicated environment with access to hospital admission and consultant expertise in the perioperative period.

There are several approaches to organising day case surgery. Some surgeons recommend discharge immediately after operation with a domiciliary visit by a specifically trained nurse on the following morning. We thought that because of the possibility of raised intraocular pressure it was safer for a senior house officer to make an examination at the first dressing five hours after operation with the option of admitting the patient overnight if necessary. This could have been to the patient's advantage in five patients in group 2, although the need for admission in two would probably have been obviated by the routine use of acetazolamide. A return visit could be made to the outpatient clinic three days later, but the five hour wait may be inconvenient and would confine the surgery to a morning list. Davies et al estimated a reduction in costs of about $4 \%$ by replacing the home visit with a conventional assessment in the outpatients department on the first postoperative day and this is now their preferred option. ${ }^{4}$ However, a visit three to five days after operation should also be recommended to screen for the development of uveitis; we subsequently think that all patients without complications may be discharged immediately after operation taking routine oral acetazolomide $500 \mathrm{mg}$ at the time of discharge, followed by a $250 \mathrm{mg}$ dose night and morning for the next 24 hours and a visit to the eye clinic three days later. Facilities should be available for inpatient admission

\begin{tabular}{lrr} 
Table 7 & Total costs $(£)$ for 100 patients in each group \\
\hline Cost & Group 1 & Group 2 \\
\hline Equipment, drugs, and disposables & 11509 & 11509 \\
Staff & 2306 & 1701 \\
Hospital stay & 22784 & 8952 \\
\hline Total & 36599 & 22162 \\
\hline
\end{tabular}


after certain surgical complications, and at the surgeon's discretion the patient may be admitted or asked to return to the clinic the following day.

When listed for surgery all patients had agreed to provide their own transport with a named friend or relative to supervise convalescence. In the event less than $2 \%$ required extended admission for social reasons (two potential group 2 patients who dropped out of the study and one group 1 patient). In the catchment area of semirural communities in North Yorkshire we believe that $80 \%$ of patients with cataracts could be treated in a day care unit, more than had been predicted, possibly because of a relatively lower proportion of social groups IV and V than in more urban areas.

Day surgery costs less per patient than inpatient treatment, but costs will probably vary in different ophthalmic units. The basic costs listed in table 4 were for simple, nonautomated techniques of nucleus extraction through a $10 \mathrm{~mm}$ wound. Small incision surgery with phacoemulsification, which has advantages of earlier visual rehabilitation and earlier return to sporting activities, would cost more because of the involvement of automated machinery.

From the difference in staff costs between the two groups there seemed little advantage in working without a senior house officer, whose cost was $£ 3.55$ per patient, but any saving must offset against the slower turnover of group 2 patients and the absence of teaching. The porter, costed at $£ 1.33$ per patient, was unnecessary for group 2 patients. The ward nurse, costed at $£ 2.11$ per patient, was superfluous for surgery but recommended by the nursing administration for group 1 patients.

However, the main contribution towards differing costs came from inpatient stay. Our figure of $£ 121.84$ correlates with that in Norwich of $£ 121.33$ for 24 hours' stay. ${ }^{4}$ The cost for group 1 patients could be reduced by altering the routine to ensure afternoon lists. This would have reduced the days in hospital by 81 ( $£ 9069)$, bringing the mean total cost per group 1 patient to $£ 275.30$. However, the cost of the group 2 patients could be reduced if a specific unit without hospital beds were to be built. Although ambulatory, our group 2 patients used a ward adjacent to an outpatient theatre suite which had facilities for general anaesthetic recovery and therefore more expensive nursing. Substituting the Norwich figure for a purpose built day unit of $£ 44.26$ per patient for our figure of $£ 87.46$ in the 94 patients not admitted would result in a mean total cost per group 2 patient of $£ 181.01$.

The final visual outcome at three to six months was not significantly different between the two groups with overall $99 \%$ seeing $6 / 12$ or better when unrelated conditions such as macular degeneration were excluded. Generally, the patients' preferred option was for day case surgery, provided that social care was adequate; most of all, patients in group 2 appreciated being able to return home at night. This option resulted in a valuable saving of resources.

We conclude that day case surgery is as safe but more cost effective than inpatient cataract surgery and should be recommended. Consultants and managers need to work together to provide facilities and staff to increase the availability of cataract surgery, so reducing waiting times and improving the quality of life for patients.

We thank $\mathrm{Mr} \mathrm{J}$ Proctor, district chief pharmacist, $\mathrm{Mr} \mathrm{J}$ Harrison, district finance officer, and $\mathrm{Mr} \mathrm{K}$ Eddon, acting district supplies officer, for helping to establishing the costs of ward stay, materials, equipment, and staff.

1 Bucher PJM. The status of European cataract surgery. European Fournal of Cataract Refractive Surgery 1990;2:96-100

2 Percival SPB, Setty SS. Cataract surgery: a prospective audit comparing local and general anaesthesia. Yorkshire Medicine 1990;3:6-8.

3 Percival SPB. Low cost surgery. In: $A$ colour atlas of len implantation. London: Wolfe Publishing, 1991;305-9.

4 Davies PD, Limacher E, Powell K. Outpatient cataract surgery 1982-1986. Eye 1987;1:728-34. 